\title{
THE FINAL FATE OF COALESCING COMPACT BINARIES: FROM BLACK HOLE TO PLANET FORMATION
}

\author{
FREDERIC A. RASIO \\ Department of Physics, \\ Massachusetts Institute of Technology, \\ Cambridge, MA 02139, USA
}

\begin{abstract}
Coalescing compact binaries are thought to be involved in a wide variety of astrophysical phenomena. In particular, they are important sources of gravitational radiation for both ground-based and space-based laser-interferometer detectors, and they may be sources of supernova explosions or gamma-ray bursts. Mergers of two white dwarfs may produce neutron stars with peculiar properties, including perhaps millisecond radio pulsars sometimes accompanied by planets (as observed in PSR 1257+12). According to a widely held belief, the coalescence of two neutron stars should produce a rapidly rotating black hole surrounded by an accretion disk or torus, but this is by no means certain. This review paper focuses on the final hydrodynamic coalescence and merger of double neutron stars and double white dwarfs, and addresses the question of the nature of the final merger products.
\end{abstract}

\section{Introduction}

The coalescence and merging of two compact stars into a single object is a very common end-point of close binary evolution. Dissipation mechanisms such as friction in common envelopes, tidal dissipation, or the emission of gravitational radiation, are always present and cause the orbits of close binary systems to decay. This review will concentrate on the coalescence of compact binaries containing either two neutron stars (hereafter NS) or two white dwarfs (WD). 


\subsection{DOUBLE NEUTRON STARS}

Many theoretical models of gamma-ray bursts (GRBs) rely on coalescing NS binaries to provide the energy of GRBs at cosmological distances (e.g., Eichler et al. 1989; Narayan, Paczyński, \& Piran 1992; Mészáros \& Rees 1992; for recent reviews see Mészáros 1999 and Piran 1999). The close spatial association of some GRB afterglows with faint galaxies at high redshifts may not be inconsistent with a NS binary merger origin, in spite of the large recoil velocities acquired by NS binaries at birth (Bloom, Sigurdsson, \& Pols 1999; but see also Bulik \& Belczynski 2000). Currently the most popular models all assume that the coalescence of two NS leads to the formation of a rapidly rotating black hole $(\mathrm{BH})$ surrounded by a torus of debris. Energy can then be extracted either from the rotation of the Kerr BH or from the material in the torus so that, with sufficient beaming, the gamma-ray fluxes observed from even the most distant GRBs can be explained (Mészáros, Rees, \& Wijers 1999). However, it is important to understand the hydrodynamic processes taking place during the final coalescence before making assumptions about its outcome. In particular, as will be argued below ( $\S 2.2)$, it is not clear that the coalescence of two $1.4 M_{\odot}$ NS forms an object that will collapse to a $\mathrm{BH}$ on a dynamical timescale, and it is not certain either that a significant amount of matter will be ejected during the merger to form an outer torus around the central object (Faber \& Rasio 2000).

Coalescing NS binaries are also important sources of gravitational waves that may be directly detectable by the large laser interferometers currently under construction, such as LIGO (Abramovici et al. 1992; see Barish \& Weiss 1999 for a recent pedagogical introduction) and VIRGO (Bradaschia et al. 1990). In addition to providing a major new confirmation of Einstein's theory of general relativity (GR), including the first direct proof of the existence of black holes (see, e.g., Flanagan \& Hughes 1998; Lipunov, Postnov, \& Prokhorov 1997), the detection of gravitational waves from coalescing binaries at cosmological distances could provide accurate independent measurements of the Hubble constant and mean density of the Universe (Schutz 1986; Chernoff \& Finn 1993; Marković 1993). Expected rates of NS binary coalescence in the Universe, as well as expected event rates in laser interferometers, have now been calculated by many groups. Although there is some disparity between various published results, the estimated rates are generally encouraging (see Kalogera 2000 for a recent review).

Many calculations of gravitational wave emission from coalescing binaries have focused on the waveforms emitted during the last few thousand orbits, as the frequency sweeps upward from $\sim 10 \mathrm{~Hz}$ to $\sim 300 \mathrm{~Hz}$. The 
waveforms in this frequency range, where the sensitivity of ground-based interferometers is highest, can be calculated very accurately by performing high-order post-Newtonian (PN) expansions of the equations of motion for two point masses (see, e.g., Owen \& Sathyaprakash 1999 and references therein). However, at the end of the inspiral, when the binary separation becomes comparable to the stellar radii (and the frequency is $\gtrsim 1 \mathrm{kHz}$ ), hydrodynamics becomes important and the character of the waveforms must change. Special purpose narrow-band detectors that can sweep up frequency in real time will be used to try to catch the last $\sim 10$ cycles of the gravitational waves during the final coalescence (Meers 1988; Strain \& Meers 1991). These "dual recycling" techniques are being tested right now on the German-British interferometer GEO 600 (Danzmann 1998). In this terminal phase of the coalescence, when the two stars merge together into a single object, the waveforms contain information not just about the effects of GR, but also about the interior structure of a NS and the nuclear equation of state (EOS) at high density. Extracting this information from observed waveforms, however, requires detailed theoretical knowledge about all relevant hydrodynamic processes. If the NS merger is followed by the formation of a $\mathrm{BH}$, the corresponding gravitational radiation waveforms will also provide direct information on the dynamics of rotating core collapse and the BH "ringdown" (see, e.g., Flanagan \& Hughes 1998).

\subsection{DOUBLE WHITE DWARFS}

Coalescing WD binaries have long been discussed as possible progenitors of Type Ia supernovae (Iben \& Tutukov 1984; Webbink 1984; Paczyński 1985; see Branch et al. 1995 for a recent review). To produce a supernova, the total mass of the system must be above the Chandrasekhar mass. Given evolutionary considerations, this requires two C-O or O-Ne-Mg WD. Yungelson et al. (1994) showed that the expected merger rate for close WD pairs with total mass exceeding the Chandrasekhar mass is consistent with the rate of Type Ia supernovae deduced from observations. Alternatively, a massive enough merger may collapse to form a rapidly rotating NS (Nomoto \& Iben 1985; Colgate 1990). Chen \& Leonard (1993) speculated that most millisecond pulsars in globular clusters might have formed in this way. In some cases planets may also form in the disk of material ejected during the coalescence and left in orbit around the central pulsar (Podsiadlowski, Pringle, \& Rees 1991). Indeed the very first extrasolar planets were discovered in orbit around a millisecond pulsar, PSR B1257+12 (Wolszczan \& Frail 1992). A merger of two magnetized WD might lead to the formation

of a NS with extremely high magnetic field, and this scenario has been proposed as a source of GRBs (Usov 1992). 
Close WD binaries are expected to be extremely abundant in our Galaxy, even though their direct detection remains very challenging (Han 1998; Saffer, Livio, \& Yungelson 1999). Iben \& Tutukov $(1984,1986)$ predicted that $\sim 20 \%$ of all binary stars produce close WD pairs at the end of their stellar evolution. More recently, theoretical estimates of the double WD formation rate in the Galaxy have converged to a value $\simeq 0.1 \mathrm{yr}^{-1}$, with an uncertainty that may be only a factor of two (Han 1998; Kalogera 2000). The most common systems should be those containing two low-mass helium WD. Their final coalescence can produce an object massive enough to start helium burning. Bailyn (1993 and references therein) and others have suggested that some "extreme horizontal branch" stars in globular clusters may be such helium-burning stars formed by the coalescence of two WD. Planets in orbit around a massive WD may also form following the binary coalescence (Livio, Pringle, \& Saffer 1992).

Coalescing WD binaries are also important sources of low-frequency gravitational waves that should be easily detectable by future space-based laser interferometers. The currently planned LISA (Laser Interferometer Space Antenna; see Folkner 1998) should have an extremely high sensitivity (down to a characteristic strain $h \sim 10^{-23}$ ) to sources with frequencies in the range $\sim 10^{-4}-1 \mathrm{~Hz}$. Han (1998) estimated a WD merger rate $\sim 0.03 \mathrm{yr}^{-1}$ in our own Galaxy. Individual coalescing systems and mergers may be detectable in the frequency range $\sim 10-100 \mathrm{mHz}$. In addition, the total number $\left(\sim 10^{4}\right)$ of close WD binaries in our Galaxy emitting at lower frequencies $\sim 0.1-10 \mathrm{mHz}$ (the emission lasting for $\sim 10^{2}-10^{4} \mathrm{yr}$ before final merging) should provide a continuum background signal of amplitude $h \sim 10^{-20}-10^{-21}$ (Hils et al. 1990). The detection of the final burst of gravitational waves emitted during an actual merger would provide a unique opportunity to observe in "real time" the hydrodynamic interaction between the two degenerate stars, possibly followed immediately by a supernova explosion, nuclear outburst, or some other type of electromagnetic signal.

\section{Coalescing Binary Neutron Stars}

\subsection{HYDRODYNAMICS OF NEUTRON STAR MERGERS}

The final hydrodynamic merger of two NS is driven by a combination of relativistic and fluid effects. Even in Newtonian gravity, an innermost stable circular orbit (ISCO) is imposed by global hydrodynamic instabilities, which can drive a close binary system to rapid coalescence once the tidal interaction between the two stars becomes sufficiently strong. The existence of these global instabilities for close binary equilibrium configurations containing a compressible fluid, and their particular importance for binary NS 
systems, were demonstrated for the first time by Rasio \& Shapiro (1992, 1994, 1995; hereafter RS1-3) using numerical hydrodynamic calculations. These instabilities can also be studied using analytic methods. The classical analytic work for close binaries containing an incompressible fluid (e.g., Chandrasekhar 1969) was extended to compressible fluids in the work of Lai, Rasio, \& Shapiro (1993a,b, 1994a,b,c, hereafter LRS1-5). This analytic study confirmed the existence of dynamical instabilities for sufficiently close binaries. Although these simplified analytic studies can give much physical insight into difficult questions of global fluid instabilities, fully numerical calculations remain essential for establishing the stability limits of close binaries accurately and for following the nonlinear evolution of unstable systems all the way to complete coalescence.

A number of different groups have now performed such calculations, using a variety of numerical methods and focusing on different aspects of the problem. Nakamura and collaborators (see Nakamura \& Oohara 1998 and references therein) were the first to perform 3D hydrodynamic calculations of binary NS coalescence, using a traditional Eulerian finite-difference code. Instead, RS used the Lagrangian method SPH (Smoothed Particle Hydrodynamics). They focused on determining the ISCO for initial binary models in strict hydrostatic equilibrium and calculating the emission of gravitational waves from the coalescence of unstable binaries. Many of the results of RS were later independently confirmed by New \& Tohline (1997) and Swesty, Wang, \& Calder (1999), who used completely different numerical methods but also focused on stability questions, and by Zhuge, Centrella, \& McMillan (1994, 1996), who also used SPH. Zhuge et al. (1996) also explored in detail the dependence of the gravitational wave signals on the initial NS spins. Davies et al. (1994) and Ruffert et al. (1996, 1997) have incorporated a treatment of the nuclear physics in their hydrodynamic calculations (done using SPH and PPM codes, respectively), motivated by models of GRBs at cosmological distances. All these calculations were performed in Newtonian gravity, with some of the more recent studies adding an approximate treatment of energy and angular momentum dissipation through the gravitational radiation reaction (e.g., Janka et al. 1999; Rosswog et al. 1999), or even a full treatment of PN gravity to lowest order (Ayal et al. 2000; Faber \& Rasio 2000).

All recent hydrodynamic calculations agree on the basic qualitative picture that emerges for the final coalescence (see Fig. 1). As the ISCO is approached, the secular orbital decay driven by gravitational wave emission is dramatically accelerated (see also LRS2, LRS3). The two stars then plunge rapidly toward each other, and merge together into a single object in just a few rotation periods. In the corotating frame of the binary, the

relative radial velocity of the two stars always remains very subsonic, so 
that the evolution is nearly adiabatic. This is in sharp contrast to the case of a head-on collision between two stars on a free-fall, radial orbit, where shock heating is very important for the dynamics (RS1; Shapiro 1998). Here the stars are constantly being held back by a (slowly receding) centrifugal barrier, and the merging, although dynamical, is much more gentle. After typically $1-2$ orbital periods following first contact, the innermost cores of the two stars have merged and a secondary instability occurs: mass shedding sets in rather abruptly. Material (typically $\sim 10 \%$ of the total mass) is ejected through the outer Lagrange points of the effective potential and spirals out rapidly. In the final stage, the spiral arms widen and merge together, forming a nearly axisymmetric thick disk or torus around the inner, maximally rotating dense core.

In GR, strong-field gravity between the masses in a binary system is alone sufficient to drive a close circular orbit unstable. In close NS binaries, GR effects combine nonlinearly with Newtonian tidal effects so that the ISCO is encountered at larger binary separations and lower orbital frequency than predicted by Newtonian hydrodynamics alone, or GR alone for two point masses. The combined effects of relativity and hydrodynamics on the stability of close compact binaries have only very recently begun to be studied, using both analytic approximations (basically, PN generalizations of LRS; see, e.g., Lai \& Wiseman 1997; Lombardi, Rasio, \& Shapiro 1997; Shibata \& Taniguchi 1997), as well as numerical calculations in 3D incorporating simplified treatments of relativistic effects (e.g., Baumgarte et al. 1998; Marronetti, Mathews \& Wilson 1998; Wang, Swesty, \& Calder 1998; Faber \& Rasio 2000).

Several groups have been working on a fully general relativistic calculation of the final coalescence, combining the techniques of numerical relativity and numerical hydrodynamics in 3D (Baumgarte, Hughes, \& Shapiro 1999; Landry \& Teukolsky 2000; Seidel 1998; Shibata \& Uryu 2000). However this work is still in its infancy, and only very preliminary results of test calculations have been reported so far.

\subsection{BLACK HOLE FORMATION}

The final fate of a NS-NS merger depends crucially on the NS EOS, and on the extraction of angular momentum from the system during the final merger. For a stiff NS EOS, it is by no means certain that the core of the final merged configuration will collapse on a dynamical timescale to form a $\mathrm{BH}$. One reason is that the Kerr parameter $J / M^{2}$ of the core may exceed unity for extremely stiff EOS (Baumgarte et al. 1998), although Newtonian and PN hydrodynamic calculations suggest that this is never the case (see, e.g., Faber \& Rasio 2000). More importantly, the rapidly rotating core may 

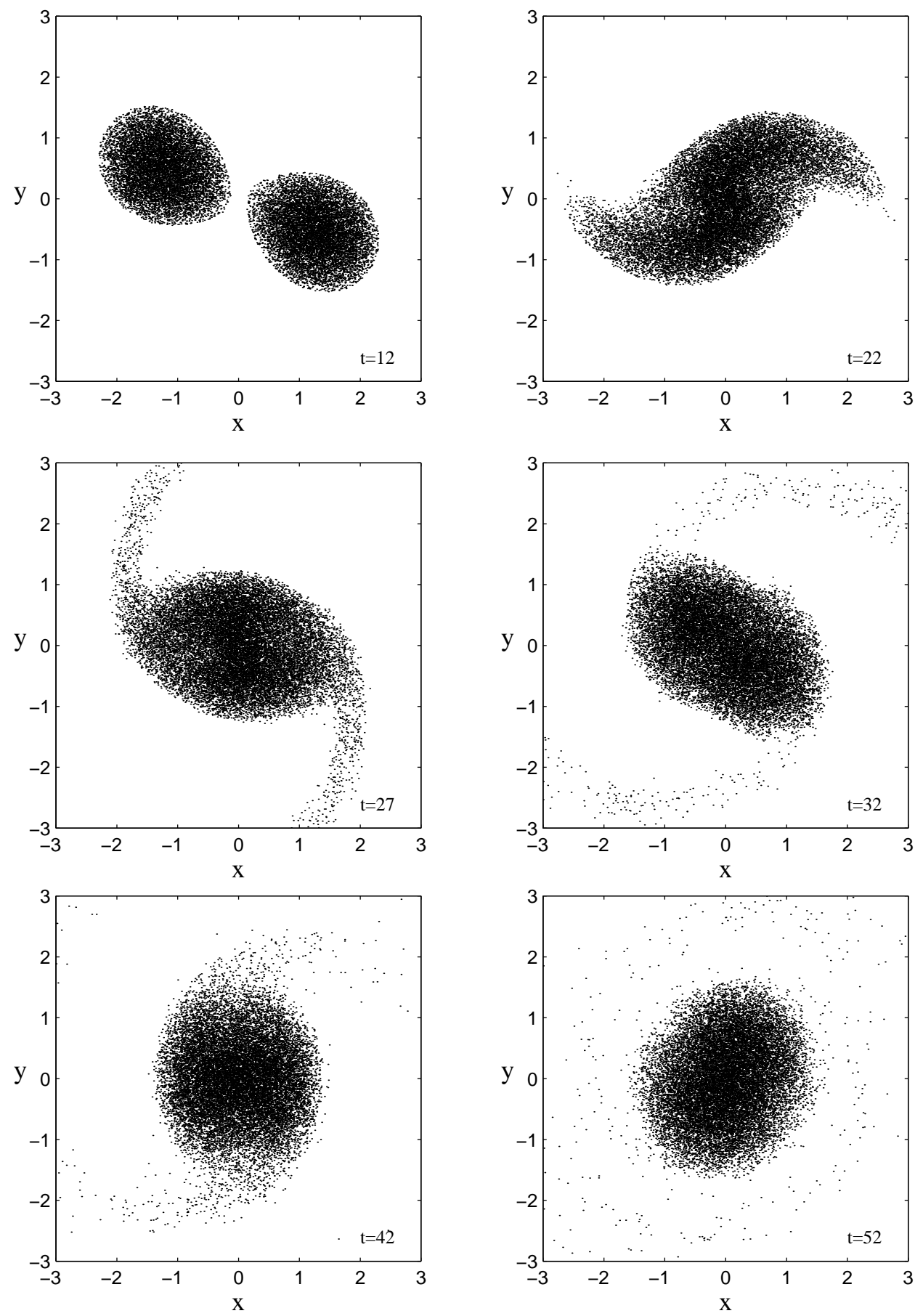

Figure 1. Post-Newtonian SPH calculation of the coalescence of two identical neutron stars modeled as simple $\Gamma=3$ polytropes. Projections of a subset of all SPH particles onto the orbital $(x-y)$ plane are shown at various times. Units are such that $G=M=R=1$ where $M$ and $R$ are the mass and radius of each star initially. The orbital rotation is counter-clockwise. From Faber \& Rasio (2000). 
in fact be dynamically stable.

Take the obvious example of a system containing two identical $1.35 M_{\odot}$ NS. The total baryonic mass of the system for a stiff NS EOS is then about $3 M_{\odot}$. Almost independent of the spins of the NS, all hydrodynamic calculations suggest that about $10 \%$ of this mass will be ejected into the outer torus, leaving at the center a maximally rotating object with baryonic mass $\simeq 2.7 M_{\odot}$ (Any hydrodynamic merger process that leads to mass shedding will produce a maximally rotating object since the system will have ejected just enough mass and angular momentum to reach its new, stable quasiequilibrium state). Most stiff NS EOS (including the well-known "AU" and "UU" EOS of Wiringa et al. 1988; see Akmal et al. 1998 for a recent update) allow stable, maximally rotating NS with baryonic masses exceeding $3 M_{\odot}$ (Cook, Shapiro, \& Teukolsky 1994), i.e., well above the mass of the final merger core. Differential rotation (not taken into account in the calculations of Cook et al. 1994) can further increase this maximum stable mass very significantly (see Baumgarte, Shapiro, \& Shibata 2000). Thus the hydrodynamic merger of two NS with stiff EOS and realistic masses is not expected to produce a BH. This expectation is confirmed by the preliminary full-GR calculations of Shibata \& Uryu (2000), for polytropes with $\Gamma=2$, which indicate collapse to a $\mathrm{BH}$ only when the two NS are initially very close to the maximum stable mass.

For slowly rotating stars, the same stiff NS EOS give maximum stable baryonic masses in the range $2.5-3 M_{\odot}$, which may or may not exceed the total merger core mass. Therefore, collapse to a $\mathrm{BH}$ could still occur on a timescale longer than the dynamical timescale, following a significant loss of angular momentum. Indeed, processes such as electromagnetic radiation, neutrino emission, and the development of various secular instabilities

(e.g., r-modes), which may lead to angular momentum losses, take place on timescales much longer than the dynamical timescale (see, e.g., Baumgarte \& Shapiro 1998, who show that neutrino emission is probably negligible). These processes are therefore decoupled from the hydrodynamics of the coalescence. Unfortunately their study is plagued by many fundamental uncertainties in the microphysics.

\subsection{THE IMPORTANCE OF THE NEUTRON STAR SPINS}

The question of the final fate of the merger could also depend crucially on the NS spins and on the evolution of the fluid vorticity during the final coalescence. Close NS binaries are likely to be nonsynchronized. Indeed, the tidal synchronization time is almost certainly much longer than the orbital decay time (Kochanek 1992; Bildsten \& Cutler 1992). For NS binaries that are far from synchronized, the final coalescence involves some new, complex 
hydrodynamic processes (Rasio \& Shapiro 1999).

Consider for example the case of an irrotational system (containing two nonspinning stars at large separation; see LRS3). Because the two stars appear to be counter-spinning in the corotating frame of the binary, a vortex sheet (where the tangential velocity jumps discontinuously by $\Delta v \sim 0.1 c$ ) appears when the stellar surfaces come into contact. Such a vortex sheet is Kelvin-Helmholtz unstable on all wavelengths and the hydrodynamics is therefore extremely difficult to model accurately given the limited spatial resolution of $3 \mathrm{D}$ calculations. The breaking of the vortex sheet generates some turbulent viscosity so that the final configuration may no longer be irrotational. In numerical simulations, however, vorticity is quickly generated through spurious shear viscosity, and the merger remnant is observed to evolve rapidly (in just a few rotation periods) toward uniform rotation.

The final fate of the merger could be affected drastically by these processes. In particular, the shear flow inside the merging stars (which supports a highly triaxial shape; see Rasio \& Shapiro 1999) may in reality persist long enough to allow a large fraction of the total angular momentum in the system to be radiated away in gravitational waves during the hydrodynamic phase of the coalescence. In this case the final merged core may resemble a Dedekind ellipsoid, i.e., it will have a triaxial shape supported entirely by internal fluid motions, but with a stationary shape in the inertial frame (so that it no longer radiates gravitational waves). This state will be reached on the gravitational radiation reaction timescale, which is no more than a few tens of rotation periods. On the (much longer) viscous timescale, the core will then evolve to a uniform, slowly rotating state and will probably collapse to a BH. In contrast, in all 3D numerical simulations performed to date, the shear is quickly dissipated, so that gravitational radiation never gets a chance to extract more than a small fraction $(\$ 10 \%)$ of the angular momentum, and the final core appears to be a uniform, maximally rotating object (stable to collapse) exactly as in calculations starting from synchronized binaries. However this behavior is most likely an artefact of the large spurious shear viscosity present in the $3 \mathrm{D}$ simulations.

In addition to their obvious significance for gravitational wave emission, these issues are also of great importance for models of GRBs that depend on energy extraction from a torus of material around the central BH. Indeed, if a large fraction of the total angular momentum is removed by the gravitational waves, rotationally-induced mass shedding may not occur at all during the merger, eventually leaving a $\mathrm{BH}$ with no surrounding matter, and no way of extracting energy from the system. Note also that, even without any additional loss of angular momentum through gravitational radiation, PN effects tend to reduce drastically the amount of matter ejected during the merger (Faber \& Rasio 2000). 


\section{Coalescing White Dwarf Binaries}

\subsection{HYDRODYNAMICS OF WHITE DWARF MERGERS}

The results of RS3 for polytropes with $\Gamma=5 / 3$ show that hydrodynamics also plays an important role in the coalescence of two WD, either because of dynamical instabilities of the equilibrium configuration, or following the onset of dynamically unstable mass transfer. Systems with mass ratios $q \approx 1$ must evolve into deep contact before they become dynamically unstable and merge. Instead, equilibrium configurations for binaries with $q$ sufficiently far from unity never become dynamically unstable. However, once these binaries reach their Roche limit, dynamically unstable mass transfer occurs and the less massive star is completely disrupted after a small number $(<10)$ of orbital periods (see also Benz et al. 1990). In both cases, the final merged configuration is an axisymmetric, rapidly rotating object with a core - thick disk structure similar to that obtained for coalescing NS (RS2, RS3; see also Mochkovitch \& Livio 1989).

\subsection{THE FINAL FATE: COLLAPSE TO A NEUTRON STAR? PLANETS?}

For two massive enough WD, the merger product may be well above the Chandrasekhar mass $M_{C h}$. The object may therefore explode as a (Type Ia) supernova, or perhaps collapse to a NS. The rapid rotation and possibly high mass (up to $\sim 2 M_{C h}$ ) of the object must be taken into account for determining its final fate. Unfortunately, rapid rotation and the possibility of starting from an object well above the Chandrasekhar limit have not been taken into account in most previous theoretical calculations of "accretioninduced collapse" (AIC), which consider a nonrotating WD just below the Chandrasekhar limit accreting matter slowly and quasi-spherically (e.g., Canal et al. 1990; Nomoto \& Kondo 1991; see Fryer et al. 1999 for a recent 2-D SPH calculation including rotation). Under these assumptions it is found that collapse to a NS is possible only for a narrow range of initial conditions. In most cases, a supernova explosion follows the ignition of the nuclear fuel in the degenerate core. However, the fate of a much more massive object with substantial rotational support and large deviations from spherical symmetry (as would be formed by dynamical coalescence) may be very different.

If a NS does indeed form, and later accretes some of the material ejected during the coalescence, a millisecond radio pulsar may emerge. Planets around this millisecond pulsar may be formed at large distances $\sim 1 \mathrm{AU}$ following the viscous evolution of the remaining material in the outer disk (Podsiadlowski, Pringle \& Rees 1991; Phinney \& Hansen 1993). This is one of the possible formation scenarios for the extraordinary planetary system 
discovered around the millisecond pulsar PSR B1257+12 (see Wolszczan 1999 for a recent update; Podsiadlowski 1993 for alternative planet formation scenarios). This system contains three confirmed Earth-mass planets in quasi-circular orbits (Wolszczan \& Frail 1992; Wolszczan 1994). The planets have masses of $0.015 / \sin i_{1} \mathrm{M}_{\oplus}, 3.4 / \sin i_{2} \mathrm{M}_{\oplus}$, and $2.8 / \sin i_{3} \mathrm{M}_{\oplus}$, where $i_{1}, i_{2}$ and $i_{3}$ are the inclinations of the orbits with respect to the line of sight, and are at distances of $0.19 \mathrm{AU}, 0.36 \mathrm{AU}$, and $0.47 \mathrm{AU}$, respectively, from the pulsar. In addition, the unusually large second and third frequency derivatives of the pulsar suggest the existence of a fourth, more distant and massive planet in the system (Wolszczan 1999). The simplest interpretation of the present best-fit values of the frequency derivatives implies a mass of about $100 / \sin i_{4} \mathrm{M}_{\oplus}$ (i.e., comparable to Saturn's mass) for the fourth planet, at a distance of about $38 \mathrm{AU}$ (i.e., comparable to Pluto's distance from the Sun), and with a period of about $170 \mathrm{yr}$ in a circular, coplanar orbit (Wolszczan 1996; Joshi \& Rasio 1997). However, if, as may well be the case, the first pulse frequency derivative is not entirely accelerationinduced, then the fourth planet can have a wide range of masses (Joshi \& Rasio 1997). In particular, it can have a mass comparable to that of Mars (at a distance of $9 \mathrm{AU}$ ), Uranus (at a distance of $25 \mathrm{AU}$ ) or Neptune (at a distance of $26 \mathrm{AU}$ ). The presence of this fourth planet, if confirmed, would place strong additional constraints on possible formation scenarios, as both the minimum mass and minimum angular momentum required in the protoplanetary disk would increase considerably (see Phinney \& Hansen 1993 for a general discussion).

\section{Acknowledgements}

This work was supported by NSF Grant AST-9618116, NASA ATP Grant NAG5-8460, and by an Alfred P. Sloan Research Fellowship. Our computational work is supported by the National Computational Science Alliance. 


\section{References}

1. Abramovici, M., et al. 1992, Science, 256, 325

2. Akmal, A., Pandharipande, V.R., \& Ravenhall, D.G. 1998, PRC, 58, 1804

3. Ayal, S., Piran, T., Oechslin, R., Davies, M.B., \& Rosswog, S. 2000, ApJ, submitted [astro-ph/9910154]

4. Bailyn, C.D. 1993, in Structure and Dynamics of Globular Clusters, eds. S.G. Djorgovski \& G. Meylan, (San Francisco: ASP Conf. Series, Vol. 50), 191.

5. Barish, B.C., \& Weiss, R. 1999, Physics Today, 52, No. 10, 44

6. Baumgarte, T.W., Cook, G.B., Scheel, M.A., Shapiro, S.L., \& Teukolsky, S.A. 1998, PRD 57, 7299

7. Baumgarte, T.W., Hughes, S.A., \& Shapiro, S.L. 1999, PRD, 60, 087501

8. Baumgarte, T.W., \& Shapiro, S.L. 1998, ApJ, 504, 431

9. Baumgarte, T.W., Shapiro, S.L., \& Shibata, M. 2000, ApJL, 528, L29

10. Benz, W., Cameron, A.G.W., Press, W.H., \& Bowers, R.L. 1990, ApJ, 348, 647

11. Bildsten, L., \& Cutler, C. 1992, ApJ, 400, 175

12. Bloom, J.S., Sigurdsson, S., \& Pols, O.R. 1999, MNRAS, 305, 763

13. Bradaschia, C., et al. 1990, Nucl. Instr. Methods A, 289, 518

14. Branch, D., Livio, M., Yungelson, L.R., Boffi, F.R., \& Baron, E. 1995, PASP, 107, 1019

15. Bulik, T., \& Belczynski, K. 2000, to appear in the Proceedings of the 5th Huntsville Gamma Ray Burst Symposium, ed. R.M. Kippen (AIP Conf. Series) [astro$\mathrm{ph} / 0001150]$

16. Canal, R., Garcia, D., Isern, J., \& Labay, J. 1990, ApJ, 356, L51

17. Chandrasekhar, S. 1969, Ellipsoidal Figures of Equilibrium (New Haven: Yale University Press); Revised Dover edition 1987

18. Chen, K, \& Leonard, P.J.T. 1993, ApJ, 411, L75

19. Chernoff, D.F., \& Finn, L.S. 1993, ApJL, 411, L5

20. Colgate, S.A. 1990, in Supernovae, ed. S.E. Woosley (New York: Springer-Verlag), 585

21. Cook, G. B., Shapiro, S. L., \& Teukolsky, S. L. 1994, ApJ, 424, 823

22. Danzmann, K. 1998, in Relativistic Astrophysics, eds. H. Riffert et al. (Proc. of 162nd W.E. Heraeus Seminar, Wiesbaden: Vieweg Verlag), 48

23. Davies, M.B., Benz, W., Piran, T., \& Thielemann, F.K. 1994, ApJ, 431, 742

24. Eichler, D., Livio, M., Piran, T., \& Schramm, D.N. 1989, Nature, 340, 126

25. Faber, J., \& Rasio, F.A. 2000, PRD, submitted [gr-qc/9912097]

26. Flanagan, E.E., \& Hughes, S.A. 1998, PRD, 57, 4566

27. Folkner, W.M. (ed.) 1998, Laser Interferometer Space Antenna, Second International LISA Symposium on the Detection and Observation of Gravitational Waves in Space, AIP Conference Proceedings, Vol. 456

28. Fryer, C., Benz, W., Herant, M., \& Colgate, S.A. 1999, ApJ, 516, 892

29. Hils, D., Bender, P.L., \& Webbink, R.F. 1990, ApJ, 360, 75

30. Iben, I., Jr., \& Tutukov, A.V. 1984, ApJ Suppl., 54, 335

31. Iben, I., Jr., \& Tutukov, A.V. 1986, ApJ, 311, 753

32. Janka, H., Eberl, T., Ruffert, M., \& Fryer, C.L. 1999, ApJ, 527, L39

33. Joshi, K.J., \& Rasio, F.A. 1997, ApJ, 479, 948

34. Kalogera, V. 2000, to appear in the Proceedings of the 3rd Amaldi Conference on Gravitational Waves, ed. S. Meshkov [astro-ph/9911532]

35. Kochanek, C.S. 1992, ApJ, 398, 234

36. Lai, D., Rasio, F.A., \& Shapiro, S.L. 1993a, ApJS, 88, 205 [LRS1]

37. Lai, D., Rasio, F. A., \& Shapiro, S.L. 1993b, ApJ, 406, L63 [LRS2]

38. Lai, D., Rasio, F. A., \& Shapiro, S.L. 1994a, ApJ, 420, 811 [LRS3]

39. Lai, D., Rasio, F. A., \& Shapiro, S.L. 1994b, ApJ, 423, 344 [LRS4]

40. Lai, D., Rasio, F. A., \& Shapiro, S.L. 1994c, ApJ, 437742 [LRS5]

41. Lai, D., \& Wiseman, A.G. 1997, PRD, 54, 3958 
42. Landry, W., \& Teukolsky, S.A. 2000, PRD, submitted [gr-qc/9912004]

43. Lipunov, V.M., Postnov, K.A., \& Prokhorov, M.E. 1997, AstL, 23, 492

44. Livio, M., Pringle, J.E., \& Saffer, R.A. 1992, MNRAS, 257, 15P

45. Lombardi, J.C., Rasio, F.A., \& Shapiro, S.L. 1997, PRD, 56, 3416

46. Marković, D. 1993, PRD, 48, 4738

47. Marronetti, P., Mathews, G.J., \& Wilson, J.R. 1998, PRD, 58, 107503

48. Meers, B.J. 1988, PRD, 38, 2317

49. Mészáros, P. 1999, Prog. Theor. Phys. Supp., 136, 78 [astro-ph/9912546]

50. Mészáros, P., \& Rees, M.J. 1992, ApJ, 397, 570

51. Mészáros, P., Rees, M.J., \& Wijers, R.A.M.J. 1999, NewA, 4, 303

52. Mochkovitch, R., \& Livio, M. 1989, A\&A, 209, 111

53. Nakamura, T., \& Oohara, K. 1998, preprint [gr-qc/9812054]

54. Narayan, R., Paczyński, B., \& Piran, T. 1992, ApJ, 395, L83

55. New, K.C.B., \& Tohline, J.E. 1997, ApJ, 490, 311

56. Nomoto, K., \& Iben, I., Jr. 1985, ApJ, 297, 531

57. Nomoto, K., \& Kondo, Y. 1991, ApJ, 367, L19

58. Owen, B.J., \& Sathyaprakash, B.S. 1999, PRD, 60, 022002

59. Paczyński, B. 1985, in Cataclysmic Variables and Low-mass X-ray Binaries, eds. D.Q. Lamb \& J. Patterson (Dordrecht: Reidel), 1

60. Phinney, E.S., \& Hansen, B.M.S. 1993, in Planets around Pulsars, ed. J.A. Phillips, S.E. Thorsett, \& S.R. Kulkarni (ASP Conf. Series Vol. 36), 371

61. Piran, T. 1999, Phys. Rep., 314, 575 [astro-ph/9907392]

62. Podsiadlowski, P., Pringle, J.E., \& Rees, M.J. 1991, Nature, 352, 783

63. Podsiadlowski, P. 1993, in Planets around Pulsars, ed. J.A. Phillips, S.E. Thorsett, \& S.R. Kulkarni (ASP Conf. Series Vol. 36), 149

64. Rasio, F.A., \& Shapiro, S.L. 1992, ApJ, 401, 226 [RS1]

65. Rasio, F.A., \& Shapiro, S.L. 1994, ApJ, 432, 242 [RS2]

66. Rasio, F.A., \& Shapiro, S.L. 1995, ApJ, 438, 887 [RS3]

67. Rasio, F.A., \& Shapiro, S.L. 1999, CQG, 16, 1

68. Rosswog, S., Liebendoerfer, M., Thielemann, F.-K., Davies, M.B., Benz, W., \& Piran, T. 1999, A\&A, in press

69. Ruffert, M., Janka, H.-T., \& Schäfer, G. 1996, A\&A, 311, 532

70. Ruffert, M., Rampp, M., \& Janka, H.-T. 1997, A\&A, 321, 991

71. Saffer, R.A., Livio, M., \& Yungelson, L.R. 1999, in 11th European Workshop on

White Dwarfs, ed. S.-E. Solheim \& E.G. Meistas (ASP Conf. Series Vol. 169), 260

72. Schutz, B.F. 1986, Nature, 323, 310

73. Seidel, E. 1998, in Relativistic Astrophysics, eds. H. Riffert et al. (Proc. of 162nd

W.E. Heraeus Seminar, Wiesbaden: Vieweg Verlag), 229

74. Shapiro, S.L. 1998, PRD, 58, 103002

75. Shibata, M. \& Taniguchi, K. 1997, PRD 56, 811

76. Shibata, M., \& Uryu, K. 2000, PRD, 61, 064001

77. Strain, K.A., \& Meers, B.J. 1991, PRL, 66, 1391

78. Swesty, F.D., Wang, E.Y.M., \& Calder, A.C. 1999, preprint [astro-ph/9911192]

79. Usov, V.V. 1992, Nature, 357, 472

80. Wang, E.Y.M., Swesty, F.D., \& Calder, A.C. 1998, in Proceedings of the Second Oak Ridge Symposium on Atomic and Nuclear Astrophysics, [astro-ph/9806022]

81. Webbink, R.F. 1984, ApJ, 277, 355

82. Wiringa, R.B., Fiks, V., \& Fabrocini, A. 1988, PRC, 38, 1010

83. Wolszczan, A. \& Frail, D.A. 1992, Nature, 355, 145

84. Wolszczan, A. 1994, Science, 264, 538

85. Wolszczan, A. 1996, in IAU Colloquium 160, Pulsars: Problems and Progress, ed. S. Johnston et al. (ASP Conf. Series Vol. 105), 91

86. Wolszczan, A. 1999, in Pulsar Timing, General Relativity and the Internal Structure of Neutron Stars, ed. Z. Arzoumanian, F. Van der Hooft, \& E.P.J. van den Heuvel (Amsterdam: Koninklijke Nederlandse Akademie van Wetenschappen), 101 
87. Yungelson, L.R., Livio, M., Tutukov, A.V., \& Saffer, R.A. 1994, ApJ, 420, 336

88. Zhuge, X., Centrella, J.M., \& McMillan, S.L.W. 1994, PRD, 50, 6247

89. Zhuge, X., Centrella, J.M., \& McMillan, S.L.W. 1996, PRD, 54, 7261 